\title{
Research on the Construction of Knowledge Map for the Course of Information Olympiad in Primary and Secondary Schools
}

\author{
G.Y. Zhang *, X.H. Wang, X.B. Shen, W.W. Bai and X. Huang \\ Capital Normal University, Beijing \\ *jkbzgy@163.com
}

\begin{abstract}
Primary and secondary information science as an engineering discipline, how to promote students' mastery of information science has become one of the most important topics in the research of information science. In this paper, the knowledge map is introduced to manage the knowledge of information science. The knowledge map of information Olympiad in primary and secondary schools is drawn by constructing the knowledge map model. It can help students understand knowledge correctly in the process of information overload by presenting the relationship between knowledge nodes, and it is used to organize and display the knowledge nodes and their relationships.
\end{abstract}

Keywords: Information Olympiad; Primary and Secondary Schools; Knowledge Map; Knowledge Node

\section{Introduction}

In the field of primary and secondary education, information science is a subject that uses computer and its programming to analyze and solve problems, while information Olympiad is one of the five international competitions which is regarded as intellectual and ability of the competition for students. ${ }^{[1]}$ National Olympiad in informatics is a national youth informatics competition and universal activities, which be approved by the State Ministry of education, science and technology of China Association ,sponsored by the Chinese Academy of computer science. This competition aim to popularize the knowledge of computer science, cultivate students' engineering quality, and promote STEM Education. Some scholars believe that STEM education contains STEM independent disciplines of learning, the key of STEM education is that students how to learn and use knowledge. ${ }^{[2]}$ In China, STEM education has a more important significance, students can master the knowledge of independent disciplines, but they lack the understanding of learning knowledge, the use of knowledge map is a good solution to this problem.

\section{Related Research}

With the development of computer information technology, knowledge has become the core power of the progress and development of human society. Knowledge map is a scientific and effective management of knowledge, so that the rapid search and sharing of knowledge has become the trend of the times. ${ }^{[3]}$ B. C. Brooks first proposed the concept of knowledge map, he believes that the knowledge map is the use of knowledge network to explain the process of knowledge change and promote the understanding of knowledge. ${ }^{[4]}$ As an important field of knowledge management, knowledge map has been paid more and more attention and played an important role. It helps the students to understand what the knowledge describes and how to find useful knowledge. ${ }^{[5]}$

\section{Knowledge Map Model}

This paper attempts to introduce the knowledge map into the construction of the information Olympiad course. Knowledge map consists of nodes and relations of knowledge, which is a structured knowledge representation. By reading the relevant literature, the author summed up the 
basic knowledge map model, as shown in Figure 1.

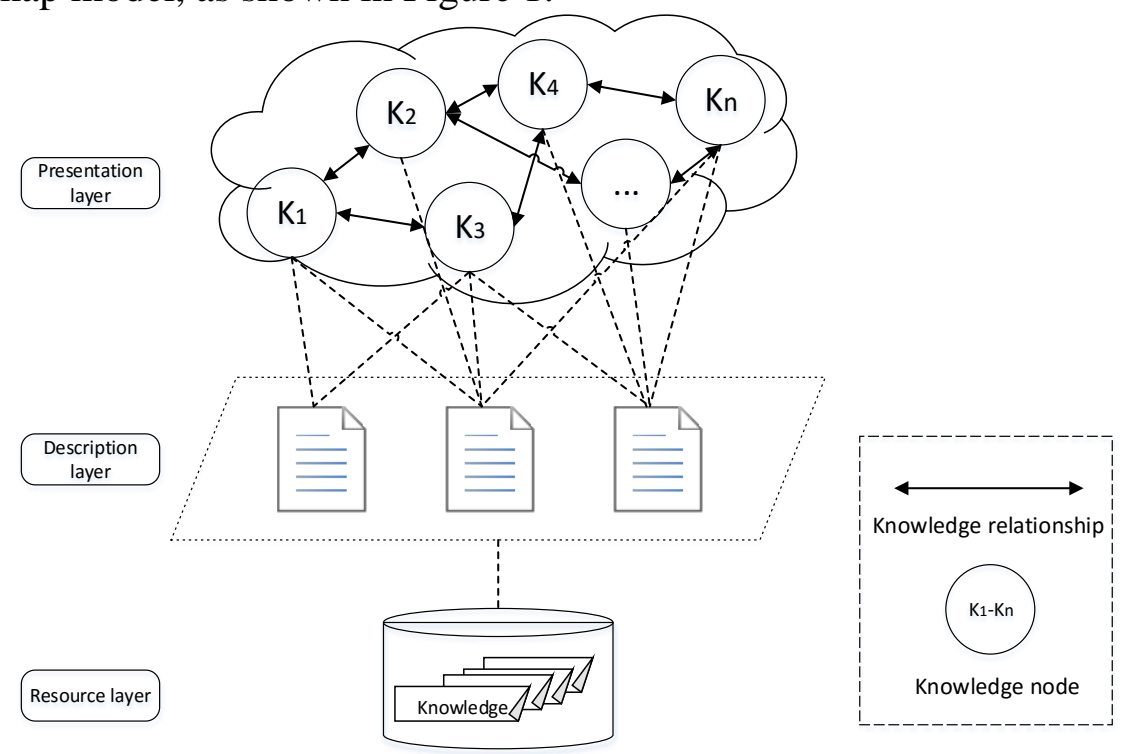

Figure 1. Knowledge Map Hierarchy Model

The knowledge map model consists of three layers, which are resource layer, description layer and presentation layer. The resource layer mainly includes the knowledge required by the syllabus and the necessary knowledge points to participate in information Olympiad; the main function of the description layer is to model the knowledge of the resource layer and abstract the main characteristics of the knowledge and describe each knowledge node involved information Olympiad knowledge; The presentation layer is mainly a visual representation of the knowledge map, and the components of the knowledge map include the relationship between the knowledge nodes, the knowledge nodes, and the visual presentation. The use of visualization of the various knowledge nodes and the relationship between the comprehensive organization and display, and thus promote the knowledge of the level and clarity, to facilitate the students to integrate knowledge, analysis and evaluation. This is also based on the problem as the core to carry out the construction of knowledge map, to solve the problem, to find knowledge of the clues to reveal the course system and knowledge.

\section{The Construction of Knowledge Map of Information Olympiad Course in Primary and Secondary Schools}

\subsection{Knowledge Identification and Organization}

The identification and organization of knowledge is mainly based on the formal method to identify the knowledge of the information science, which is based on the knowledge node, the attributes of knowledge nodes and the relationship between knowledge nodes. In this paper, we consider the National Olympiad in Informatics in Provinces (NOIP) as the research object. In the outline of NOIP League, it includes six main contents: the basic knowledge about computer, the basic operation of the computer, the basic knowledge of program design, data structure, program design, the algorithm processing. ${ }^{[6]}$ The architecture of knowledge information (as shown in Figure 2) to sort out the knowledge and the relation for the need of students to participate in NOIP. The design of knowledge system classify the knowledge, take out the visual representation of knowledge by the tree structure chart, take the information Olympiad as the root node, which based on basic computer knowledge, basic computer operation, basic knowledge of programming second layer nodes, gradually extended down. 


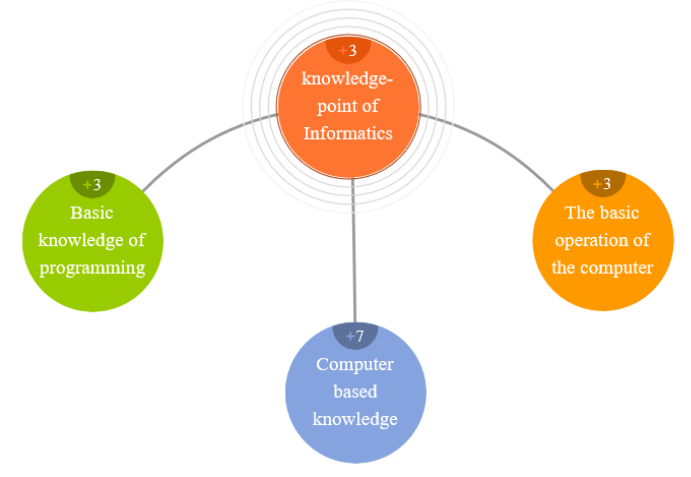

Figure 2. The system of Course of Information Olympiad in Primary and Secondary Schools

\subsection{Knowledge Arrangement}

The arrangement of knowledge is the visualization of the knowledge that students must have and should have, and based on the course knowledge system as a blueprint knowledge to sort out. At the same time, it also gives a comprehensive summary and generalization of the knowledge of the course. In Figure 2, the knowledge system of primary school information Olympiad is taken as a reference to sort out the knowledge involved in NOIP. This process is not only the finishing of the whole course of knowledge, but also the refinement of the relationship between the various knowledge, and ultimately sort out the hierarchy between knowledge. As shown in Figure 3, with the information Olympiad knowledge point as the root node, the basic knowledge of the computer, the basic operation of the computer, the basic knowledge of programming and other knowledge as the second node and so on, step by step, the knowledge points involved in the syllabus are fully displayed, which is also a kind of understanding and comprehension of the field of knowledge.

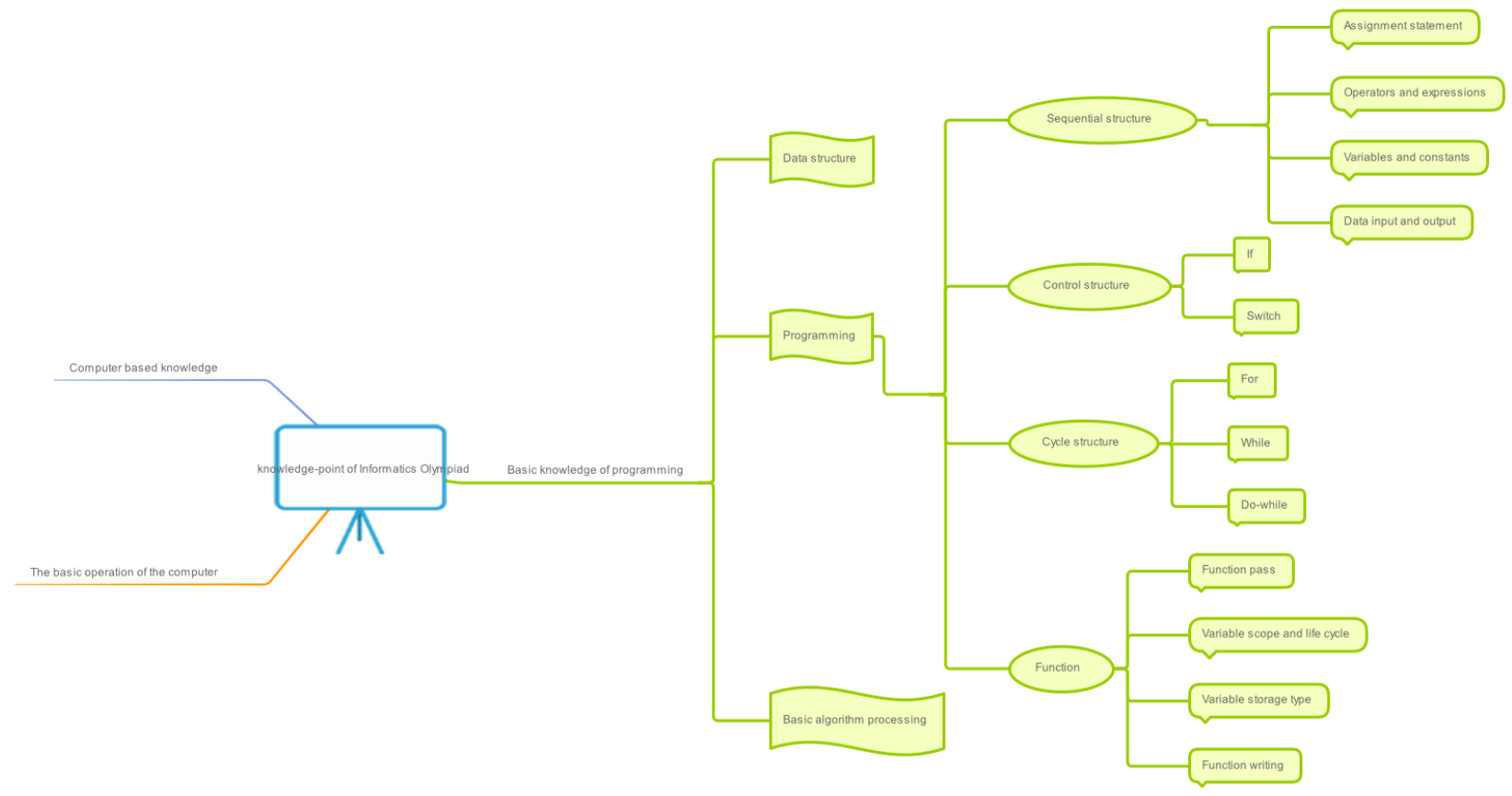

Figure 3. Some Knowledge Points in the Course of Information Olympiad in Primary and Secondary Schools

\subsection{Knowledge Relation}

Although there are many relationships between knowledge nodes, we comb the relationship between the characteristics of each node element from the perspective of relationship between 
ordering node. The knowledge node can be divided into several levels: the knowledge node does not have the precedence relationship of knowledge, which is the parallel relationship. Regardless of the time, Such kind of knowledge can be carried out at the same time, and do not affect each other; The upper level knowledge node is the predecessor node of the next level knowledge node, the next level knowledge node is the successor node of the upper level knowledge node, the prior knowledge must be mastered before learning the knowledge, the successor knowledge is the extension and expansion of the pre knowledge. Therefore, we must grasp the predecessor knowledge before learning the successor knowledge and not exchange order. The knowledge nodes $\mathrm{K}=\left\{K_{1}, K_{2}, K_{3}\right.$. $\left.\cdot K_{n}\right\} . \mathrm{n}$ is the total number of knowledge nodes. Constructing the knowledge node relation matrix as follows ${ }^{[7]}$

$$
\mathrm{R}=\left[\begin{array}{cccc}
R\left(K_{1}, K_{1}\right) & R\left(K_{1}, K_{2}\right) & \ldots & R\left(K_{1}, K_{n}\right) \\
\ldots & \ldots & \ldots \\
R\left(K_{m}, K_{1}\right) & R\left(K_{m}, K_{2}\right) & R\left(K_{m}, K_{n}\right)
\end{array}\right] .
$$

$\mathrm{R}\left(K_{\mathrm{m}}, K_{n}\right)$ represents the relationship between knowledge $K_{\mathrm{m}}$ node and $K_{n}$ node. If $\mathrm{R}\left(K_{\mathrm{m}}, K_{n}\right)=$ 0 , the $K_{\mathrm{m}}$ node and $K_{n}$ node is not related; If $\mathrm{R}\left(K_{\mathrm{m}}, K_{n}\right)=1$, the $K_{\mathrm{m}}$ node and $K_{n}$ node is the parallel relation. If $\mathrm{R}\left(K_{\mathrm{m}}, K_{n}\right)=2$, the $K_{\mathrm{m}}$ node and $K_{n}$ node is the predecessor and successor relation. In other words, the $K_{\mathrm{m}}$ node is the precursor knowledge of $K_{n}$ node, the $K_{n}$ node is the successor knowledge of the $K_{\mathrm{m}}$ node.

\section{The Application of Knowledge Map in the Course of Information Olympiad in Primary and Secondary Schools}

Information science is an independent subject in primary and secondary school course, which involves a wide range of knowledge. It is not conducive to the establishment of students' own knowledge system in the form of catalogue.

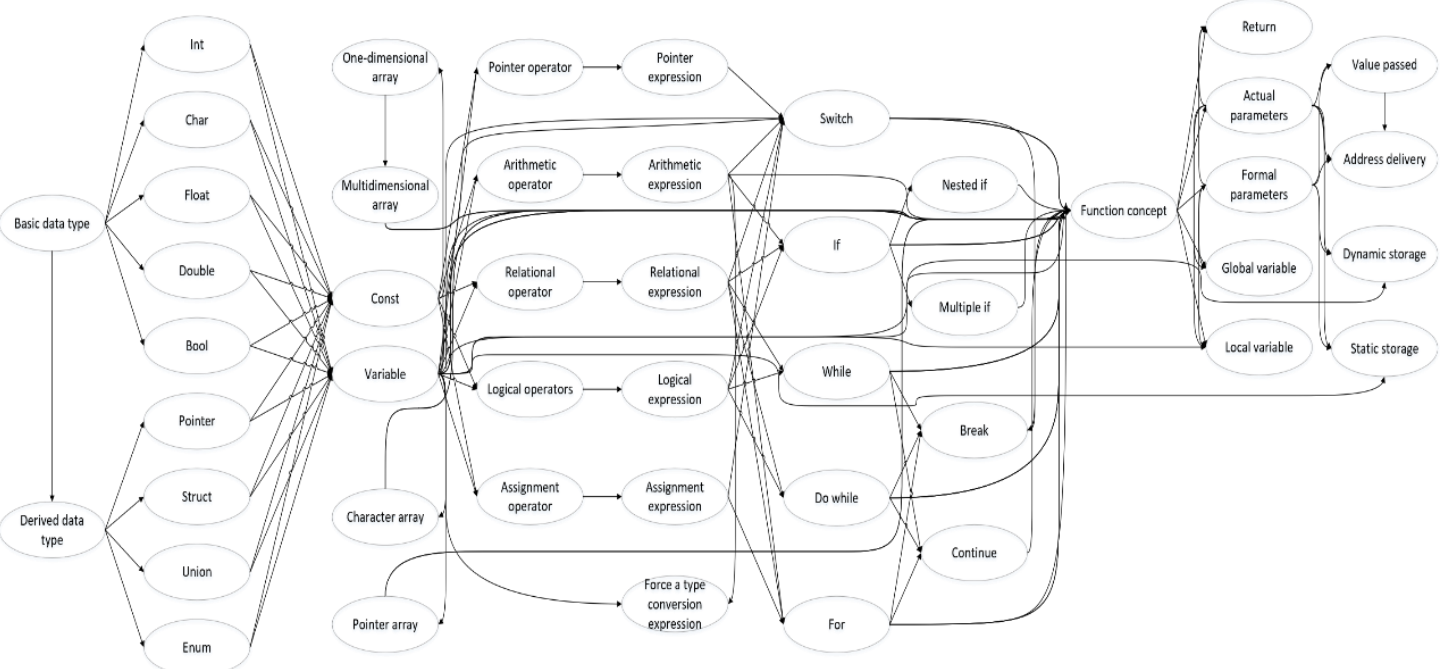

Figure 4. The knowledge Map of Information Olympiad Course in Primary and Secondary Schools

Taking into account the characteristics of information science knowledge and the overall cognitive level of primary and secondary school students, the information Olympiad knowledge is visualized by the knowledge map, which enables the students to understand the knowledge structure of the course and organize to memory the knowledge of information Olympiad quickly. According to the internal logical relationship between the knowledge points of the course, the knowledge map establishes the successor relation between the knowledge nodes, which is helpful for the students to understand the course better and promote the mastery of knowledge. The characteristics of 
knowledge map visualization can be used as a knowledge assessment tool to evaluate the individual's knowledge and help the teacher to diagnose the learning situation of the learners. As a result of the description of knowledge map on the relationship between the entire knowledge systems, once learners have problems with learning a point of knowledge, they can quickly find the pre-knowledge point that lead to the problem through the knowledge map, which allows teachers to have targeted counseling. Learners can also use knowledge maps to guide the acquisition of knowledge, to learn the next knowledge points, and finally to form the knowledge network. The knowledge map of the information Olympiad course in primary and secondary schools is shown in figure 4.

\section{Conclusions}

The knowledge map overcome the knowledge out of order situation, clearly shows the relationship between knowledge and knowledge through the knowledge map, is an effective tool for knowledge management. When the students solve a problem, by observing the knowledge map, the students can obtain the relationship between the knowledge system and knowledge nodes, and promote the use and understanding of knowledge. In this paper, the knowledge map is applied to the course of information Olympiad, manage knowledge, reveal the relationship between the knowledge of knowledge nodes, and make course knowledge is no longer a single individual, but the relationship between each other in an orderly whole. To show the knowledge structure in a visualized way, the learners can understand the knowledge at a glance. But in the process of building a knowledge map, to find the learning process of tacit knowledge and visual representation, in a more effective way to promote the students of knowledge and memory, understanding, application, analysis, evaluation and creation, is still a worthy of further study, and is the focus of the next step of the research work question. With the development of digital technology, the function and effect of knowledge map will be improved and strengthened constantly.

\section{References}

[1] Zhian Xu. Cultivating Students' Information Literacy in Information Science [J]. The Chinese Journal of ICT in Education, 2012, 10:43-47.

[2] Qian Fu and Pengfei Liu. From Verification to Creation-A Research on STEM Education Performance Models in Middle and Primary Schools [J]. China Educational Technology, 2016, 04:71-78+105.

[3] Yufang Ma and Sijing Wu. Constructing the Knowledge Map of College Foreign Language Teaching and Research Institutions [J]. China Educational Technology, 2010, 08:88-91.

[4] Xinning Su etc. Organizational Knowledge Management [M] Beijing:National Defense Industry Press, 2004.

[5] Balaid A, Rozan M Z A, Hikmi S N, et al. Knowledge maps: A systematic literature review and directions for future research [J]. International Journal of Information Management, 2016, 36(3):451-475.

[6] Information on http://www.noi.cn/about/rules/61-2008-12-26-07-48-48

[7] Tao Huang, Feng Shi and Huali Yang. The Research of the Knowledge Map Model and the Application in Learning Resources Navigation [J]. China Educational Technology, 2015, 07: 73-78+95. 